\title{
Resistive switching and optical properties of strontium ferrate titanate thin film prepared via chemical solution deposition
}

\author{
Jun LI, Xingui TANG*, Qiuxiang LIU, Yanping JIANG, Zhenxun TANG \\ School of Physics and Optoelectric Engineering, \\ Guangdong University of Technology, Guangzhou 510006, China
}

Received: September 17, 2020; Revised: April 10, 2021; Accepted: April 12, 2021

(c) The Author(s) 2021.

\begin{abstract}
The polycrystalline strontium ferrate titanate $\left(\mathrm{SrFe}_{0.1} \mathrm{Ti}_{0.9} \mathrm{O}_{3}, \mathrm{SFTO}\right)$ thin films have been successfully prepared by chemical solution method. By analyzing the current-voltage $(I-V)$ characteristics, we discuss the conduction mechanism of SFTO. It is found that the number of oxygen vacancy defects is increased by Fe ion doping, making SFTO be with better resistive switching property. Fe ion doping can also enhance the absorption of strontium titanate to be exposed to visible light, which is associated with the change of energy band. The band gap width $(2.84 \mathrm{eV})$ of SFTO films is figured out, which is less than that of pure strontium titanate. Due to more oxygen vacancy defects caused by Fe ion doping, the band gap width of strontium titanate was reduced slightly. The defect types of SFTO thin films can be determined by electron paramagnetic resonance spectroscopy. In addition, we analyzed the energy band and state density of SFTO by first-principles calculation based on density functional theory, and found that Fe ion doping can reduce the band gap width of strontium titanate with micro-regulation on the band structure. A chemical state of SFTO was analyzed by X-ray photo electron spectroscopy. At the same time, the structure and morphology of SFTO were characterized by X-ray diffraction and scanning electron microscope. This study deepens further understanding of the influence of Fe ion doping on the structure and properties of strontium ferrate titanate, which is expected to be a functional thin film material for memristor devices.
\end{abstract}

Keywords: $\mathrm{SrTiO}_{3}$; thin films; resistive switching; oxygen vacancy; first principles

\section{Introduction}

During the past few decades, a large number of experimental and theoretical studies on $\mathrm{ABO}_{3}$ perovskite compounds have been carried out, especially the typical material $\mathrm{SrTiO}_{3}$. One motive force to study $\mathrm{SrTiO}_{3}$ perovskite is the opportunity to regulate and control its electronic and ionic defect structures, so as to gain a wide range

\footnotetext{
* Corresponding author.

E-mail: xgtang@gdut.edu.cn
}

of applications (energy harvesting, memory device, oxygen sensor, catalysis, fuel cell cathodes, etc.) [1-5]. There are a number of researches by first-principles studies of defective perovskite to investigate electronic structure and performance [6-11]. As for $\mathrm{SrTiO}_{3}$, it can be changed into a p-type material by substituting $\mathrm{Fe}$ to Ti ions [7]. Some previous work revealed the interesting properties such as ferroelectric [8], photochromic [9], oxygen sensitive properties $[10,11]$, as well as Jahn-Teller distortion [12]. In addition, dielectric properties, defect state, and conduction mechanism have been studied in strontium ferrate titanate system [13-16]. 
In recent years, strontium ferrate titanate has attracted more and more attention in studying defect chemistry, carrier transport properties, and ferroelectric $[17,18]$. Compared with the pure $\mathrm{SrTiO}_{3}$, defect state like oxygen vacancies in strontium ferrate titanate sustains charge balance due to the diverse valence states between $\mathrm{Fe}$ and Ti ions. The formation of vacancies and defect state affects both electrical and magnetic behaviors [19]. As the previous report said, the potential barrier height is influenced as a result of oxygen vacancy concentration and chemisorption of $\mathrm{O}^{-}$and $\mathrm{O}^{2-}$ at the grain boundary [20]. Fe ion doping of $10 \%$ content improves the ferroelectric of $\mathrm{SrTiO}_{3}$ exactly. Moreover, ferromagnetic properties in perovskites depend on not only oxygen vacancies and defects but also the annealing atmosphere and temperature [21]. Afterward, the annealing atmosphere effects on device characteristics have been investigated [22,23].

Up to now, iron-substituted $\mathrm{SrTiO}_{3}$ system has been studied by multifarious experimental and theoretical researches. Nevertheless, there is still not any thorough elucidation of the relation to the concentration of point defects. An intensive understanding of the energy band regulation-property relation is essential. In this work, we presented the preparation and electric properties including resistive switching characteristic, ferroelectric, and optoelectronic of $\mathrm{SrFe}_{0.1} \mathrm{Ti}_{0.9} \mathrm{O}_{3-\sigma}$ thin film deposited on fluorine doped tin oxide (FTO) coated glass substrate via chemical solution deposition method. In this study, it is the first time to utilize the first-priciples theoretical calculation based on density functional theory (DFT) for investigating the electronic structure and state density of perovskite material $\mathrm{SrFe}_{x} \mathrm{Ti}_{1-x} \mathrm{O}_{3-\sigma}$ with $10 \% \mathrm{Fe}$ ions to elaborate the experimental results, which can give us enlightenment to explore and verify the effect of doping in band gap regulation.

\section{Experimental}

The method we used in our work to prepare the $\mathrm{SrFe}_{0.1} \mathrm{Ti}_{0.9} \mathrm{O}_{3-\sigma}$ (SFTO) thin film on the FTO (the thickness is $330 \mathrm{~nm}$ and the resistance is $7 \Omega$ ) coated glass substrate (purchased from the School Experimental Raw Materials Purchasing Platform, Guangdong University of Technology) was chemical solution deposition (CSD) [24]. The raw materials we selected were strontium acetate $(0.859 \mathrm{~g})$, iron 9-hydrate nitrate $(0.161 \mathrm{~g})$, and butyl titanate $(1.225 \mathrm{~mL})$. In addition, methanol, and $36 \%$ acetic acid and acetylacetone were selected as solvent and stabilizer, respectively. First, strontium acetate was dissolved in methanol and iron nitrate was dissolved in $36 \%$ acetic acid. In order to dissolve the strontium acetate and iron 9-hydrate nitrate completely, we put the beaker on the heating table and stirred it for $2 \mathrm{~h}$ to be dissolved at a temperature of $50{ }^{\circ} \mathrm{C}$. Secondly, appropriate amount of butyl titanate was taken from the measuring cylinder, and 2 drops (about $0.04 \mathrm{~mL}$ ) of acetylacetone were added to stabilize it. Finally, we mixed the three solutions together and bottled the mixed solution after filtering. The concentration of the final mixed solution was $0.2 \mathrm{~mol} / \mathrm{L}$. In order to ensure the quality of the prepared thin film, we put the prepared solution stand at room temperature for one day and observed that there was no precipitate before casting the film. FTO-coated glass substrates were cleaned completely before the preparation. We put the FTO-coated glass substrates into ultrasonic cleaner for $15 \mathrm{~min}$ and dried the substrates on the heating platform. The above operation needs to be repeated three times to ensure that the substrates are thoroughly cleaned. The solution was dropped onto the FTO substrate and spun at a speed of $4000 \mathrm{rpm}$ for $30 \mathrm{~s}$. Two high speed spins were performed according to the thickness of the required film. After that, thin film samples were baked on a heating table at $400{ }^{\circ} \mathrm{C}$ for $30 \mathrm{~min}$. Hydrofluoric acid with low concentration will not corrode the FTO layer. Therefore, we used the hydrofluoric acid $(0.1 \mathrm{~mol} / \mathrm{L})$ to wipe one corner on the SFTO layer of the thin film samples - in order to plate the electrode on the surface after annealing. At the end, all the thin film samples (SFTO and STO) were annealed at $650{ }^{\circ} \mathrm{C}$ for $15 \mathrm{~min}$ in the oxygen atmosphere by the rapid thermal annealing furnace RTP-1000D4 facility.

$\mathrm{X}$-ray diffraction, X-ray photoelectron spectroscopy (XPS), and electron paramagnetic resonance spectroscopy were used to characterize the structure, chemical states, and defect center of the thin film samples. The currentvoltage $(I-V)$ characteristic of the $\mathrm{Au} / \mathrm{SFTO} / \mathrm{FTO} / \mathrm{Glass}$ device was measured with the two-probe method by the Keithley 2400 programmable electrometer under room temperature. Additionally, first-principles calculations based on density functional theory were used to analyze the band structure and electronic states of the SFTO samples.

\section{Results and discussion}

\section{1 Structure, morphology, and chemical states}

To prove the crystalline state of the strontium ferrate 
titanate thin film we prepared, X-ray diffraction was applied to characterize the structure of the samples. As we can seen from Fig. 1, the red and blue curves represent the XRD results of SFTO and pure strontium titanate (STO was prepared with SFTO at the same time and same conditions), respectively. It can be seen from the periodic table of chemical elements that the ionic radius of iron is smaller than that of titanium. According to the Bragg diffraction equation $(2 d \sin \theta=n \lambda)$, the XRD diffraction peaks of SFTO shift slightly to the direction of small angle. Peaks of $23.3^{\circ}(100), 32.5^{\circ}(110), 46.6^{\circ}$ (200), and $58.2^{\circ}(211)$ crystal planes of SFTO were observed obviously which are corresponding to JCPDS No. 35-0734 from the Joint Committee on Power Diffraction Standards (JCPDS) database, indicating that SFTO thin film is crystallized in cubic structure. No second phase was observed in the X-ray diffraction result. Therefore, $\mathrm{Fe}$ ions successfully replace $\mathrm{Ti}$ ions in strontium titanate matrix and the same perovskite structure of strontium ferrite as strontium titanate is formed [25]. The scanning electron microscopy (SEM) result of cross section is shown in Fig. 2(a). As can be seen from the results of the cross section image (Fig. 2(a)), the stratification is obvious. The top layer is the strontium ferrate titanate thin film, while the middle layer is FTO conductive layer, the bottom layer is the glass, the thickness of SFTO film is about $120 \mathrm{~nm}$, and the particles are relatively dense [26]. Figure 2(b) shows the morphology observed by the atomic force microscopy (AFM). From Fig. 2(b), we can see the slight sharp grain morphology with the typical surface roughness which is closely related to the annealing temperature (SFTO was treated at the high temperature of $650{ }^{\circ} \mathrm{C}$ ).

In order to analyze the chemical composition, proportion, and chemical valence of SFTO thin film sample, XPS results are shown in Fig. 3. Narrow spectrum scanning was acquired from the XPS spectra for the $\mathrm{Sr} 3 \mathrm{~d}$, Ti $2 \mathrm{p}$, $\mathrm{O} 1 \mathrm{~s}$, and $\mathrm{Fe} 2 \mathrm{p}$ levels of $\mathrm{SrFe}_{0.1} \mathrm{Ti}_{0.9} \mathrm{O}_{3-\sigma}$ thin film sample. Two peaks of $\mathrm{Sr} 3 \mathrm{~d}$ locating at $132.10 \mathrm{eV}\left(\mathrm{Sr} 3 \mathrm{~d}_{5 / 2}\right)$ and $133.78 \mathrm{eV}\left(\mathrm{Sr} 3 \mathrm{~d}_{3 / 2}\right)$ are observed in Fig. 3(a), which indicates $\mathrm{Sr}$ ions with chemical state of $2^{+}$[27]. Two peaks of Ti $2 p_{3 / 2}$ and Ti $2 p_{1 / 2}$ are located at 457.52 and $463.24 \mathrm{eV}$ in Fig. 3(b), which indicates $\mathrm{Ti}$ ions with chemical state of $4^{+}$. The $\mathrm{O} 1 \mathrm{~s}$ spectra are shown in Fig. 3(c), and the binding energy of $\mathrm{O} 1 \mathrm{~s}$ spectra possesses two peaks. The stronger peak appears at $528.59 \mathrm{eV}$ and it is closely related to $\mathrm{O}^{2-}$ ions which is associated with $\mathrm{O}$ element in SFTO lattice [4]. The lower peak at $530.54 \mathrm{eV}$ is owing to intermediate oxidation state for $\mathrm{O}$ element. It may be related to the chemical-adsorbed oxygen on the thin film surface, which is associated with defects such as oxygen vacancies. As shown in Fig. 3(d), Fe 2p XPS spectra contain a doublet of $\mathrm{Fe} 2 \mathrm{p}_{3 / 2}$ and $2 \mathrm{p}_{1 / 2}$. The positions of the peaks are located at 709.60 and $723.25 \mathrm{eV}$, respectively. It indicates Fe element with chemical state of $3^{+}$[28]. Due to spin-orbit coupling, Fe $2 p_{3 / 2}$ peak is stronger than $\mathrm{Fe} 2 \mathrm{p}_{1 / 2}$ peak. $\mathrm{Fe}^{2+}$ electronic configuration is $3 \mathrm{~d}^{6}$, while $\mathrm{Fe}^{3+}$ is $3 \mathrm{~d}^{5}$. It means that the $\mathrm{Fe}^{2+}$ would have a longer life-time in the comparison to $\mathrm{Fe}^{3+}$, and therefore the selected full width at half maximum (FWHM) of Fe $2 \mathrm{p}_{1 / 2}$ peak is expected to be smaller than the $\mathrm{Fe} 2 \mathrm{p}_{3 / 2}$ peak.

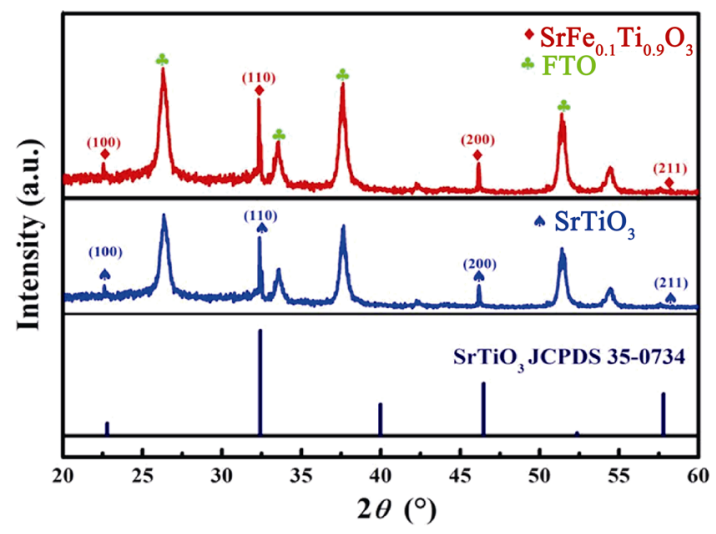

Fig. 1 XRD patterns of the SFTO film on FTO/Glass substrates.
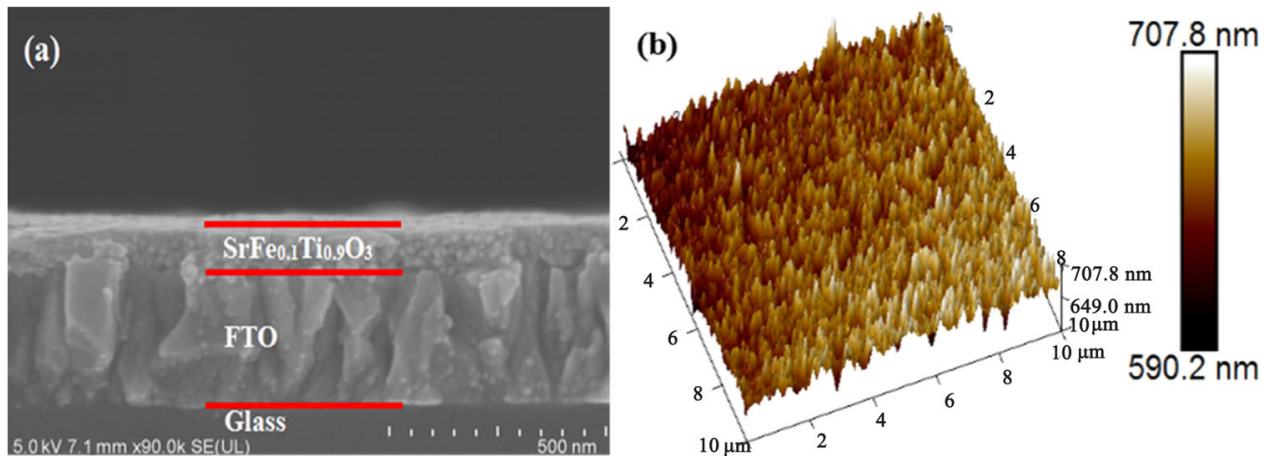

Fig. 2 (a) Cross section SEM image and (b) AFM image of the SFTO/FTO/Glass device. 

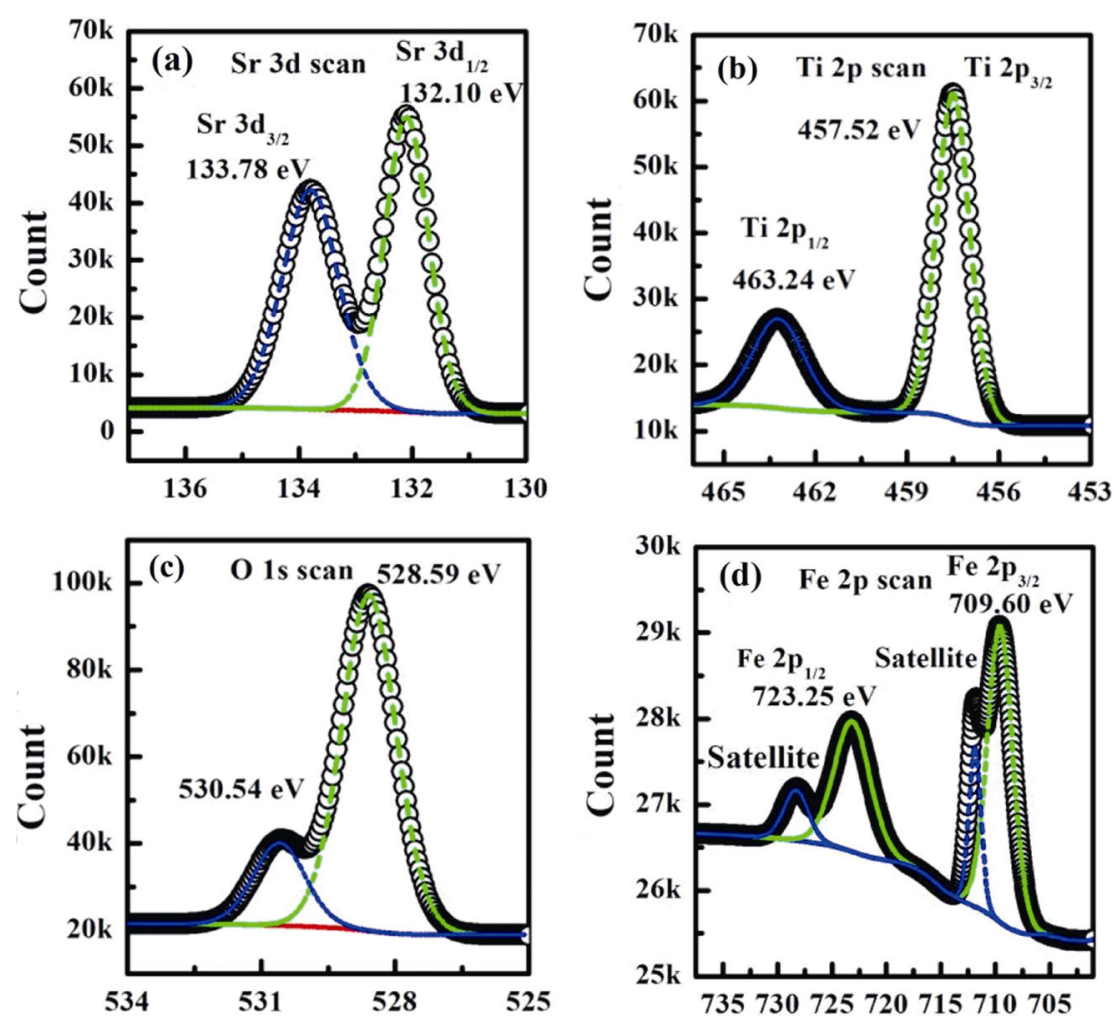

Fig. 3 XPS survey of the $\mathrm{SrFe}_{0.1} \mathrm{Ti}_{0.9} \mathrm{O}_{3-\sigma}$ thin film and the fitted narrow sweeping results of (a) $\mathrm{Sr} 3 \mathrm{~d}$, (b) Ti 2p, (c) O 1s, and (d) Fe 2p.

In addition, the bimodal spectra of $\mathrm{Fe} 2 \mathrm{p}$ indicate $\mathrm{Fe}$ element with $4^{+}$valence. Therefore, both $\mathrm{Fe}^{3+}$ and $\mathrm{Fe}^{4+}$ ions exist in SFTO thin film [29]. It has been reported in Ref. [30] that the increase of charge at the Fe site leads to the shifting of $\mathrm{Fe} 2 \mathrm{p}$ spectrum toward higher binding energy. Thus, it can be concluded that $\mathrm{SrTiO}_{3}$ with $10 \% \mathrm{Fe}$ ion doping can increase $\mathrm{Fe}^{3+}$ and $\mathrm{Fe}^{4+}$ ions effectively, resulting in the formation of oxygen vacancy defects [30]. The process can be briefly written into the defect reaction equations to deepen the understanding of $\mathrm{Fe}$ ion doping effect. The reactions are as following [31]:

$$
\begin{gathered}
2 \mathrm{O}_{\mathrm{O}}^{x} \rightarrow 2 \mathrm{~V}_{\ddot{\mathrm{O}}}+4 \mathrm{e}^{\prime}+\mathrm{O}_{2} \\
2 \mathrm{Fe}^{4+}+2 \mathrm{e}^{\prime} \rightarrow 2 \mathrm{Fe}^{3+} \\
2 \mathrm{Fe}^{3+}+2 \mathrm{e}^{\prime} \rightarrow 2 \mathrm{Fe}^{2+}
\end{gathered}
$$

where $\mathrm{e}^{\prime}$ is the free electron, $\mathrm{O}_{\mathrm{O}}^{x}$ represents the oxidation of lattice oxygen, and $\mathrm{V}_{O}$ is the oxygen vacancy.

\section{3. $2 I-V$ characteristics}

Resistive switching behavior of $\mathrm{Au} / \mathrm{SFTO} / \mathrm{FTO} / \mathrm{Glass}$ device is shown in Fig. 4. Voltage sweeping $(0 \rightarrow-3 \rightarrow$ $0 \rightarrow+3 \rightarrow 0 \mathrm{~V})$ is employed for $I-V$ measurement. It can be seen from Fig. 4(b) that the current of the Au/SFTO/
FTO/Glass device increases with the gradual increase of negative voltage at the beginning, and the device is in a low resistance state (LRS), as shown in path 1 in the Fig. 4(b). When the negative bias exceeds the threshold voltage of $2.8 \mathrm{~V}$, the current rapidly decreases from $1.7 \times 10^{-2}$ to $6 \times 10^{-4} \mathrm{~mA}$, and the state of the device switches from LRS to high resistance state (HRS). As the negative bias decreases, the current approaches 0 , as shown in path 2 in Fig. 4(b). When the scanning voltage is from 0 to $3 \mathrm{~V}$, the current of the thin film device gradually increases from 0 . When the voltage exceeds the forward threshold voltage of $2.95 \mathrm{~V}$, the current drops rapidly, and the device switches from LRS to HRS, as shown in path 3 in Fig. 4(b). When the forward bias gradually decreases from 3 to $0 \mathrm{~V}$, the current intensity also decreases to 0 , returning to the original state. At this point, a complete cycle test is completed. It can be concluded that the SFTO thin film device has the resistance switching behavior and the performance of memristors. Under the same test conditions, the resistive switching characteristics of $\mathrm{SrTiO}_{3}$ cannot be found. We will discuss the reasons in the following sections. Figure 4(c) shows the $I-V$ curve under semilogarithmic coordinates. The 100 -cycle $I-V$ curves are shown in Fig. S1 in the Electronic Supplementary Material 
(ESM). We can calculate the switch ratio from Fig. 4(c). Switch ratio $\left(R_{\text {High }} / R_{\text {Low }}\right)$ at $-2.8 \mathrm{~V}$ is about 30 . We also tested the switch retention characteristics to ensure the resistive switching stability of the strontium ferrate titanate. The cycle endurance results are shown in Figs. 5(a) and 5(b). Resistances of HRS and LRS are read at $-2.8 \mathrm{~V}$ and $+2.8 \mathrm{~V}$. Average ratio of HRS/LRS are about 30 and 10, respectively. From the results we can conclude that the resistive switching property of strontium ferrate titanate has not only good stability but also higher switching ratio compared with the pure strontium titanate.

In order to figure out the resistive switching mechanism of $\mathrm{Au} / \mathrm{SFTO} / \mathrm{FTO} / \mathrm{Glass}$ device, $I-V$ characteristics under negative and positive applied bias are replotted as $\log I$ vs. $\log V$ and shown in Figs. 6(a) and 6(b), respectively. The slopes of fitting curve in LRS under negative and positive applied bias are 1.05 and 1.09, respectively, corresponding to the ohmic conduction mechanism. For HRS, ohmic conduction mechanism is dominant in low voltage region under both negative and positive applied bias, which is confirmed by the slopes of fitting curves of 1.05 and 1.07, respectively. However, conduction mechanism switches to space-charge limited current (SCLC) mechanism in high voltage region, which is confirmed by the slopes of fitting curves of 1.93 and 2.13. In general, SCLC mechanism is mainly related to the presence of defect centers. The current density of space-charge limited current $J_{\mathrm{SCLC}}$ can be explained as following [32]:

$$
J_{\mathrm{SCLC}}=\frac{9 V^{2}}{8 d^{3}} \varepsilon \mu
$$

where $V$ is the bias voltage, $d$ is the thickness of the thin film, $\varepsilon$ is the dielectric constant of the thin film, and $\mu$ is the electron mobility. Under the application of bias voltage, the defect centers can capture the free carriers excited by the electric field. In order to understand the conduction mechanism of strontium ferrate titanate thin film devices more intuitively, a reasonable model is proposed to explain the role of oxygen vacancy in the conduction process shown in Fig. 7.
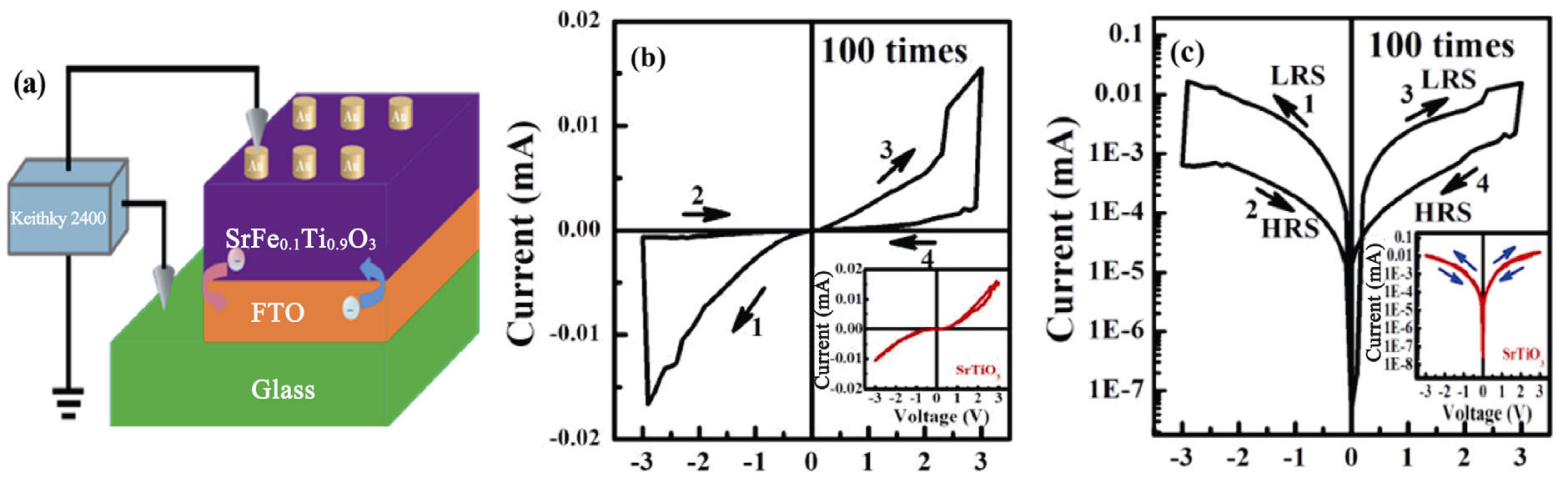

Fig. 4 Resistance properties of the SFTO/FTO/Glass device. (a) Measurement schematic diagram of the Au/SFTO/FTO/Glass device. (b) $I-V$ characteristic curve in Cartesian coordinates at the bias voltage of $\pm 3 \mathrm{~V}$ and $I-V$ characteristics measuring structure is shown in the inset. The inset is the $I-V$ characteristic of pure $\mathrm{SrTiO}_{3}$ in Cartesian coordinates. (c) $I-V$ characteristic curve in logarithmic coordinates and the direction of measurement is shown in the order of arrows from 1 to 4 . The inset is the $I-V$ characteristic of pure $\mathrm{SrTiO}_{3}$ in logarithmic coordinates.
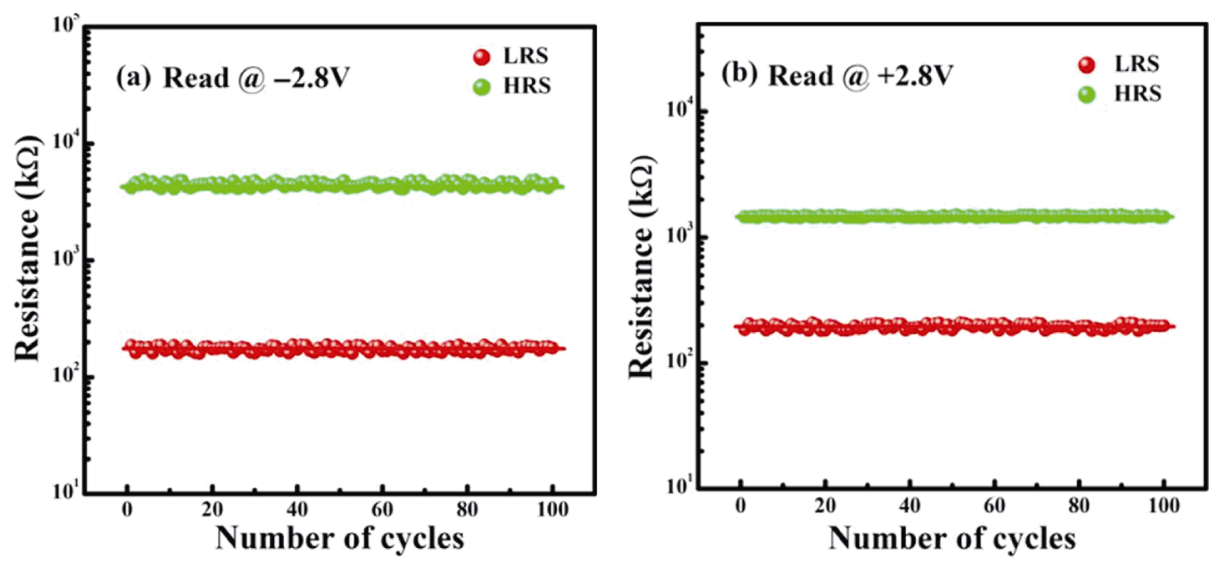

Fig. 5 Resistance retention characteristics at: (a) -2.8 and (b) $+2.8 \mathrm{~V}$ of the SFTO/FTO/Glass device. 

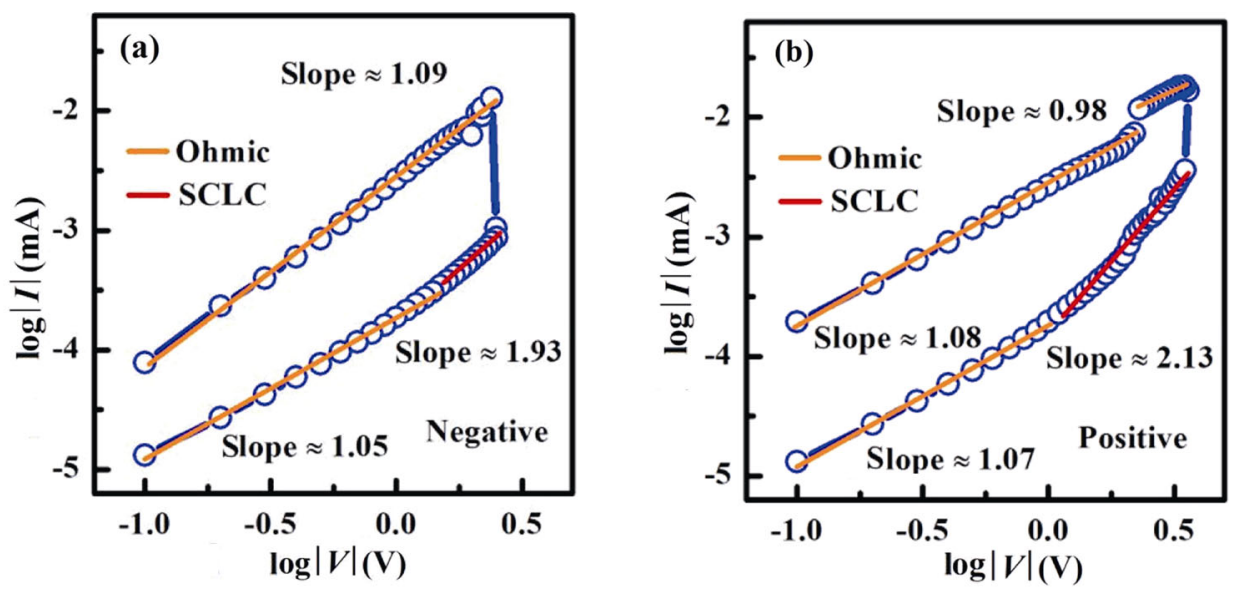

Fig. 6 Analysis of the conductance mechanism under (a) negative and (b) positive voltage bias fitted by the double logarithm.

(a)

As-prepared

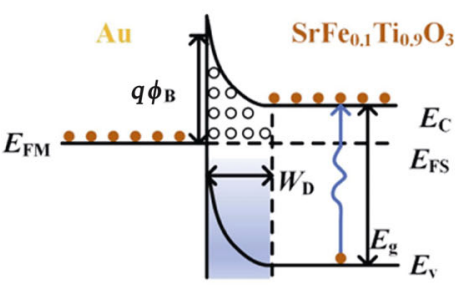

- Electrons

- Oxygen vacancies (b)

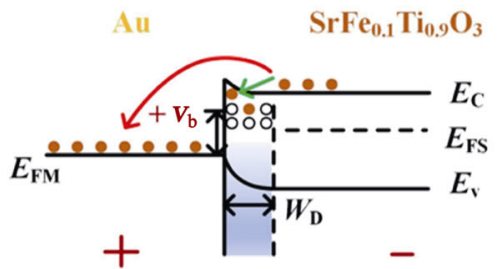

$\rightarrow$ Electron migration

$\longrightarrow$ Trap capture (c)

HRS

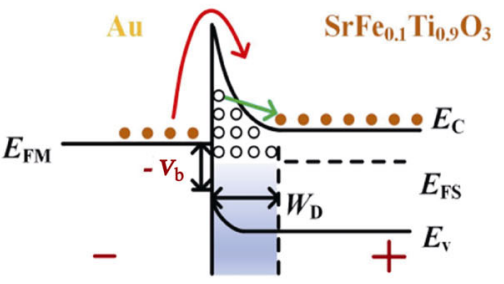

$\rightarrow$ Electron migration

$\rightarrow$ Trap release

Fig. 7 Schematic diagram of the resistance switching phenomenon of Au/SFTO/FTO/Glass device based on the Schottky barrier.

Oxygen vacancies are inevitable in the preparation of thin films and the redistribution of electric charge on the surface of the devices is generated when the electrodes are plated to form the structure of the strontium ferrate titanate thin film devices. Therefore, before the device structure is formed without bias voltage, due to different work functions of the metal electrode and the strontium ferrate titanate thin film, the two different materials contact at the interface and form the Schottky junction. The electrons cannot freely cross the Schottky barrier, and the Fermi level of the gold electrode is at the same level with the Fermi level of the strontium ferrate titanate thin film which is shown in Fig. 7(a). $E_{\mathrm{C}}$ is the conduction band in the SFTO band structure, $E_{\mathrm{V}}$ is the valence band, and $E_{\mathrm{FS}}$ is the Fermi level. $W_{\mathrm{D}}$ represents the barrier width formed by the contact between gold electrode and SFTO film. When the forward bias voltage is added, the potential barrier at the interface will decrease, the Fermi energy level of the strontium ferrate titanate thin film will rise, and the electrons will fill into the oxygen vacancy and be captured by the oxygen vacancy. A large number of electrons tend to move toward the direction of the gold electrode, forming an LRS, as shown in Fig. 7(b). When the reverse bias voltage is applied, the barrier at the interface will increase, the oxygen vacancy will release the captured electrons, and the Fermi energy level of the film will decrease. However, due to the increase of the barrier, only a small part of the electrons on the surface of the gold electrode can cross the barrier and enter the conduction band of the semiconductor thin film, thus forming an HRS which is shown in Fig. 7(c). When the direction of electric field is constantly changed, the strontium ferrate titanate thin film device can switch between high and low resistance states. Additionally, the more oxygen vacancies the thin film has, the higher ratio of high and low resistance states the thin film would have. By doping Fe ions, the defect concentration of strontium ferrate titanate thin film can be changed to produce more oxygen vacancies which affect the migration of the free electrons. Thus, by contrasting the $I-V$ characteristics between the strontium ferrate titanate thin film and the pure strontium titanate thin film (Figs. 4(b) and 4(c)) and the conduction mechanism simulation diagram analysis (Fig. 7), we can draw the conclusion that strontium ferrate titanate thin film has relatively favorable resistive switching performance 
due to the increase of oxygen vacancies.

\section{3 Optical properties}

UV-Vis absorption spectra of SFTO thin film are shown in Fig. 8(a). We utilize UV-Vis spectrophotometer (ThermoFisher Evolution 220) to measure absorption spectrum of SFTO and STO. Before the measurement, we use blank sample (which provided from the measurement system) for calibration. After that, we choose the measurement mode of reflectance. The wavelength range is from 330 to $600 \mathrm{~nm}$. In order to ensure the accuracy of reflection spectra, we repeat the measurement three times for each sample. We also utilize the same UV-Vis spectrophotometer to measure the transmittance. The test wavelength range keeps the same with the reflection measurement. Transmittance of SFTO and STO thin film rises with the increase of test wavelength. It is noted that the reflection and transmittance measurement on different thin film samples must be calibrated respectively. We repeat the transmittance measurement three times for each sample as well for attaining accuracy results. At last, we use the reflection spectra and transmission spectrum of SFTO and STO thin film samples to calculate the absorption spectrum respectively. From Fig. 8(a), It can be seen that SFTO thin film absorbs more UV light with a wavelength lower than $365 \mathrm{~nm}$, and the UV response is relatively sensitive. According to Tauc's law, the relationship between absorption coefficient $(\alpha)$ and photon energy $(h v)$ can be expressed as

$$
\begin{gathered}
\alpha=(1 / d) \times \ln (1 / T) \\
h v=1240 / \lambda
\end{gathered}
$$

where $T$ and $\lambda$ are the transmittance and wavelength, respectively. The optical band gap $\left(E_{\mathrm{g}}\right)$ can be derived by the tangent line of the known curve. $E_{\mathrm{g}}$ of $2.84 \mathrm{eV}$ is obtained for SFTO thin film as shown in Fig. 8(b), being lower than the $E_{\mathrm{g}}$ of pure $\mathrm{SrTiO}_{3}(3.24 \mathrm{eV})$. The doping of Fe ions can lower the $E_{\mathrm{g}}$ of strontium titanate. Thus, SFTO thin films have better electric properties which are expected for the application of photodetector with faster response [33].

\section{4 Electron paramagnetic resonance spectroscopy of SFTO and STO}

In order to determine the defect type of the thin film of strontium titanate with $10 \% \mathrm{Fe}$ iron doping, we studied its electron paramagnetic resonance (EPR) spectra. EPR is a magnetic resonance technique used to characterize the unpaired electrons or single electron states of materials. The relative intensity of SFTO is three times larger than that of the pure $\mathrm{SrTiO}_{3}$. In fact, there are intrinsic defects (such as oxygen vacancies and line defect) in pure $\mathrm{SrTiO}_{3}$ crystal structure and the defect states would produce defect energy level in the band gap which can trap the electrons during the electron transition process. From the EPR spectra, $g$ factor of the sample can also be calculated and used to determine whether there is an electron defect center or a whole defect center by comparing it with the $g$ factor of the free electron. Some researchers used EPR technology to analyze $\mathrm{O}^{2-}$ ions and $\mathrm{Ti}^{3+}$ ions on the surface of polycrystalline $\mathrm{TiO}_{2}$ and $\mathrm{SrTiO}_{3}$, respectively [34,35]. Figure 9 shows the EPR results of SFTO thin film sample. It is found that the EPR peak occurs at a position of $3800 \mathrm{Gs}$ magnetic field intensity. The $g$ factor of strontium titanate SFTO thin film sample can be calculated by the following equation:

$$
g=0.7144773 \times v / B_{0}
$$

where $v$ is the frequency and $B_{0}$ is the magnetic field. $g$ factor of SFTO thin film sample is 1.9237 , which is
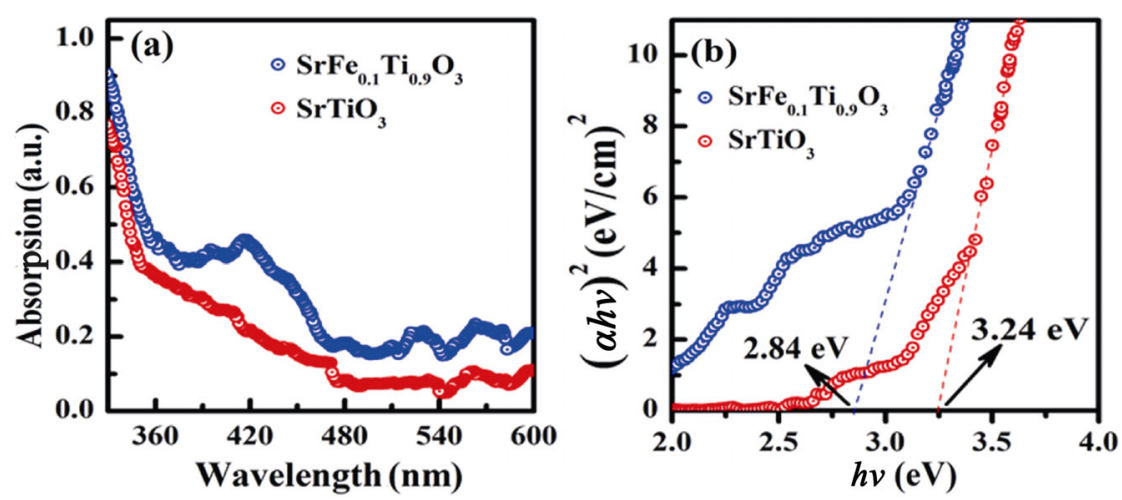

Fig. 8 Comparison of optical properties between Fe doped strontium titanate and pure strontium titanate. (a) UV-Vis absorption spectrum with the wavelength range from 330 to $600 \mathrm{~nm}$. (b) The calculated band gap results are the points at which the tangent line intersects the horizontal axis according to the Tauc's law. 


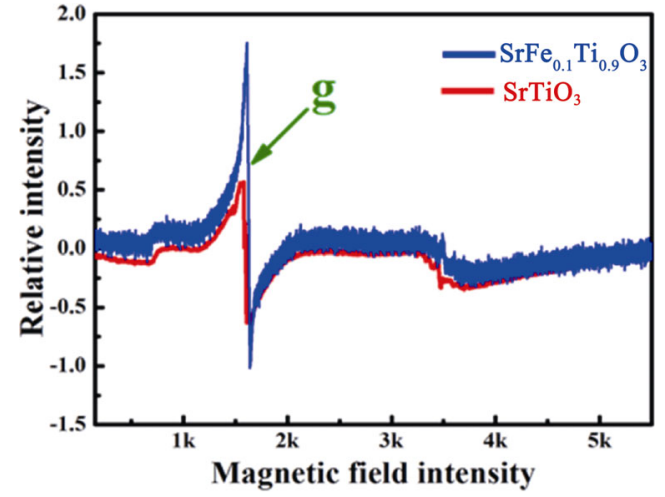

Fig. 9 EPR spectroscopy of strontium titanate films with iron content of $10 \%$. The defect type can be determined by calculating $g$ from the EPR spectroscopy.

less than that of free electron (2.0023), indicating that there is an electron defect center in the thin film sample. To some extent, it can be concluded that Fe ions have successfully replaced the Ti ions in strontium titanate matrix. Fe ions can attract more electron density distribution than $\mathrm{Ti}$ ions and lead to the redistribution of electron cloud density near the Fe-doped impurity, which is closely related to the formation of oxygen vacancies.

\section{5 First-principles calculations}

It has been reported that a phenomenon of Jahn-Teller distortion in strontium ferrate titanate was found [17].
It is closely related to the $\mathrm{Fe}-\mathrm{O}$ bond length. $\mathrm{Fe}-\mathrm{O}$ bond length will change when $\mathrm{Fe}$ ions substitute to $\mathrm{Ti}$ ions, producing relative displacement of oxygen and related oxygen defects [36]. The experiments show that Jahn-Teller distortion decreases with the increase of Fe ion concentration. Further analysis on electronic state density, band structure, and absorption spectrum can be studied via first-principles calculation based on density functional theory for SFTO. In order to better illustrate the band structure and electron state density of $\mathrm{SrFe}_{0.1} \mathrm{Ti}_{0.9} \mathrm{O}_{3-\sigma}$, $\mathrm{SrTiO}_{3-\sigma}$ and $\mathrm{SrFeO}_{3-\sigma}$ are also studied. First-principles calculations are carried out via the Vienna $a b$ initio simulation package (VASP) utilizing the projector augmented waves (PAW) technique [37]. Before the calculation based upon the density functional theory (DFT), the $k$-point grid size and the advolution are set up with respect to the cut of energy. We ensure that each structure (referring to $\mathrm{SrFe}_{0.1} \mathrm{Ti}_{0.9} \mathrm{O}_{3-\sigma}, \mathrm{SrTiO}_{3-\sigma}$, and $\mathrm{SrFeO}_{3-\sigma}$ ) has a appropriate number of $k$ points for the integration of Brillouin-zone. The electronic energies are converged to at least $10^{-5} \mathrm{eV} /$ atom. As for the modeling of SFTO ( $10 \% \mathrm{Fe}$ atoms on the Ti site), it is important to build an appropriate crystalline structure which is shown in Fig. S1 in the ESM. Calculation results are shown in Fig. 10. Among them, Figs. 10(a) and 10(b) show the band structure and electron state density of $\mathrm{SrTiO}_{3-\sigma}$, respectively. The top of the valance band and the bottom
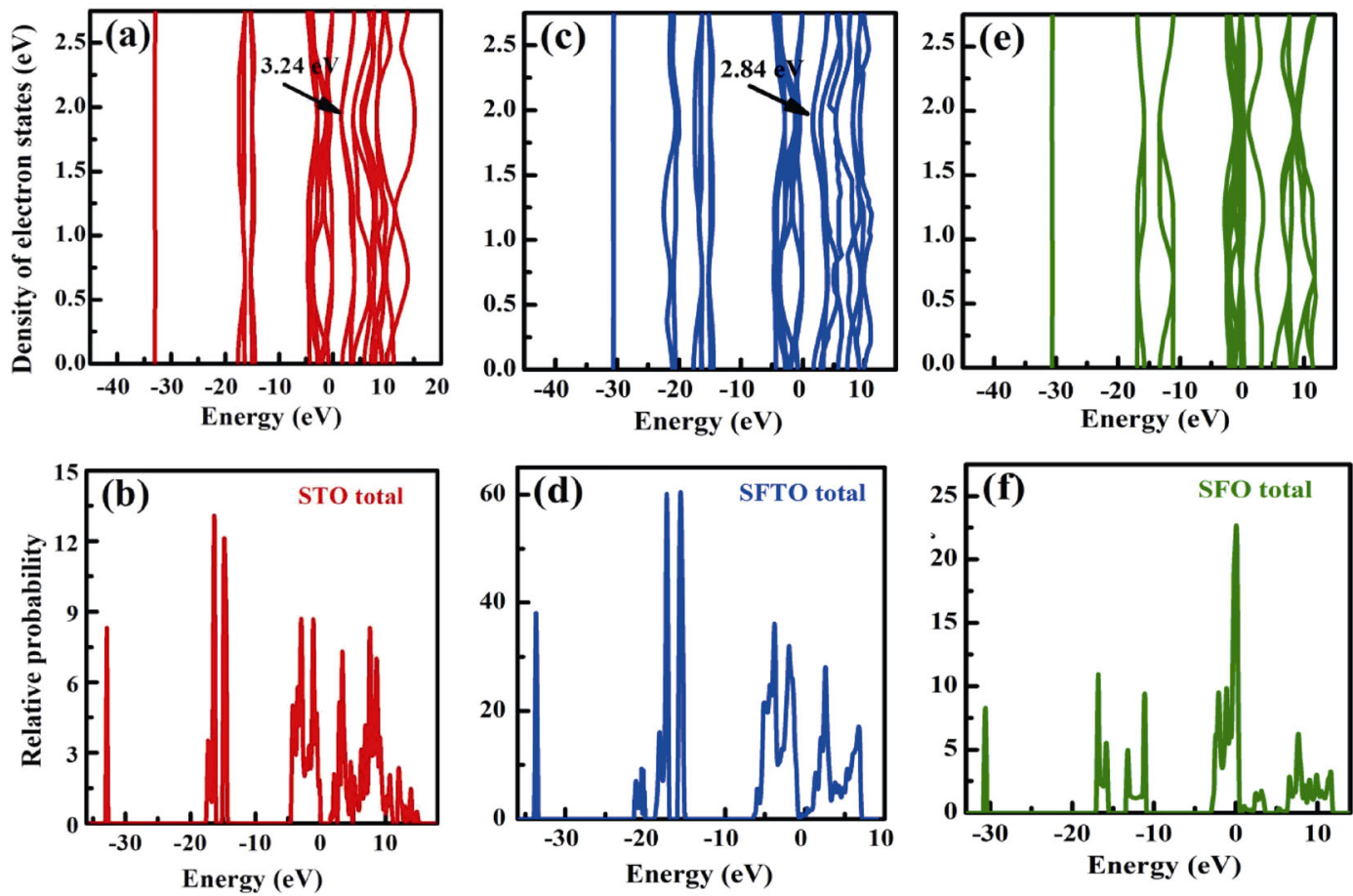

Fig. 10 Calculated band structures and density of electron states for (a, b) $\mathrm{SrTiO}_{3}$, (c, d) $\mathrm{SrFe}_{0.1} \mathrm{Ti}_{0.9} \mathrm{O}_{3-\sigma}$, and (e, f) $\mathrm{SrFeO}_{3}$. In all cases, the presentation of the valence band and conduction band is concluded. 
of conduction band are mainly made up of $\mathrm{O} 2 \mathrm{p}$ states and Ti 3d states, respectively. Figures 10(e) and 10(f) illustrate the band structure and electron state density of $\mathrm{SrFeO}_{3-\delta}$. It is obviously seen from the comparison of the electron state density for spinning up and spinning down of the electron densities, where the effect of spin polarization is derived from the Fe $2 p$ states, a little bit from Fe 3d states. The results in Figs. 10(e) and 10(f) also indicate that there is a strong superposition of $\mathrm{Fe}$ $3 \mathrm{~d}$ orbital electron states and $\mathrm{O} 2 \mathrm{p}$ orbital electron states near the Fermi level. In addition, the contribution of the main electron states near the Fermi level is derived from the $\mathrm{Eg}$ states of the $\mathrm{Fe}$ ions and $\mathrm{O} 2 \mathrm{p}$ electron states. Figures 10(c) and 10(d) show the band structure and electron state density of $\mathrm{SrFe}_{0.1} \mathrm{Ti}_{0.9} \mathrm{O}_{3-\sigma}$. The top of the valence band consists of $\mathrm{O} 2 \mathrm{p}$ orbital electron states, while the bottom of virtual bands mainly originates from Ti $3 \mathrm{~d}$ electron states [38]. It can be concluded that the band gap of strontium titanate with $10 \%$ Fe-doping is smaller than that of the pure strontium titanate, which indicates that $\mathrm{Fe}$ ions can regulate the energy band of strontium titanate and form the defect state effectively.

\section{Conclusions}

In this study, the resistance characteristics and band structure of strontium ferrate titanate/FTO devices are studied. Polycrystalline strontium ferrate titanate thin films were successfully prepared on FTO/Glass substrate by chemical solution deposition. XRD results showed that the characteristic peaks of strontium ferrite titanate were consistent with that of polycrystalline strontium titanate. It can be seen from SEM that the grains of strontium ferrate titanate films are relatively dense and have good crystallinity. The analysis results of EPR and XPS show that $\mathrm{Fe}$ ions can produce oxygen vacancy and other defects, and form electron trap center, which has important influence on the resistance characteristics of strontium ferrate titanate. Typical bipolar resistance is easy to be found in the $I-V$ characteristic test. By the means of $\log$-log fitting, we found that ohmic conduction is the conduction mechanism in the LRS, and ohmic conduction and space charge limited current conduction are the two mechanisms in the HRS. The first-principles calculation shows that the band structure of strontium titanate has changed after the doping of $\mathrm{Fe}$ ions. In addition, through the absorption spectrum test of strontium ferrate titanate, it was found that the absorption of partial visible light was enhanced. By absorption spectrum calculation, the band gap width $E_{\mathrm{g}}$ of the sample was $2.84 \mathrm{eV}$, slightly smaller than the band gap width $3.24 \mathrm{eV}$ of strontium titanate. The experimental results were consistent with the theoretical calculation.

\section{Acknowledgements}

This work was supported by the National Natural Science Foundation of China (Grant No. 11574057), the Guangdong Basic and Applied Basic Research Foundation (Grant No. 2021A1515012607), and the Science and Technology Program of Guangdong Province of China (Grant No. 2017A010104022).

\section{Electronic Supplementary Material}

Supplementary material is available in the online version of this article at https://doi.org/10.1007/s40145-021-0483-0.

\section{References}

[1] Cho S, Jang JW, Zhang WR, et al. Single-crystalline thin films for studying intrinsic properties of $\mathrm{BiFeO}_{3}-\mathrm{SrTiO}_{3}$ solid solution photoelectrodes in solar energy conversion. Chem Mater 2015, 27: 6635-6641.

[2] Janousch M, Meijer GI, Staub U, et al. Role of oxygen vacancies in Cr-doped $\mathrm{SrTiO}_{3}$ for resistance-change memory. Adv Mater 2007, 19: 2232-2235.

[3] Menesklou W, Schreiner HJ, Härdtl KH, et al. High temperature oxygen sensors based on doped $\mathrm{SrTiO}_{3}$. Sens Actuat B: Chem 1999, 59: 184-189.

[4] Kawasaki S, Nakatsuji K, Yoshinobu J, et al. Epitaxial Rh-doped $\mathrm{SrTiO}_{3}$ thin film photocathode for water splitting under visible light irradiation. Appl Phys Lett 2012, 101: 033910 .

[5] Wang L, Merkle R, Mastrikov YA, et al. Oxygen exchange kinetics on solid oxide fuel cell cathode materials - General trends and their mechanistic interpretation. J Mater Res 2012, 27: 2000-2008.

[6] Alexandrov VE, Maier J, Evarestov RA. Ab initio study of $\mathrm{SrFe}_{x} \mathrm{Ti}_{1-x} \mathrm{O}_{3}$ : Jahn-Teller distortion and electronic structure. Phys Rev B 2008, 77: 075111.

[7] Higuchi T, Tsukamoto T, Sata N, et al. Electronic structure of p-type $\mathrm{SrTiO}_{3}$ by photoemission spectroscopy. Phys Rev B 1998, 57: 6978-6983.

[8] Sarin N, Mishra M, Gupta G, et al. Deciphering the role of oxygen vacancies on structural, electrical, and magnetic properties of Fe-substituted strontium titanate. Phys Status Solidi B 2018, 255: 1700683.

[9] Berney RL, Cowan DL. Photochromism of three photosensitive Fe centers in $\mathrm{SrTiO}_{3}$. Phys Rev B 1981, 23: $37-50$. 
[10] Merkle R, Maier J. Oxygen incorporation into Fe-doped

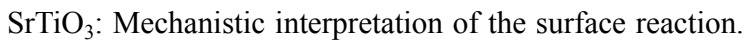
Phys Chem Chem Phys 2002, 4: 4140-4148.

[11] Rothschild A, Litzelman SJ, Tuller HL, et al. Temperatureindependent resistive oxygen sensors based on $\mathrm{SrTi}_{1-x} \mathrm{Fe}_{x} \mathrm{O}_{3-\delta}$ solid solutions. Sens Actuat B: Chem 2005, 108: 223-230.

[12] Evarestov R, Blokhin E, Gryaznov D, et al. Jahn-Teller effect in the phonon properties of defective $\mathrm{SrTiO}_{3}$ from first principles. Phys Rev B 2012, 85: 174303.

[13] Ang C, Yu Z, Jing Z, et al. Dielectric spectra and electrical conduction in Fe-doped $\mathrm{SrTiO}_{3}$. Phys Rev B 2000, 61: 3922-3926.

[14] Muenstermann R, Menke T, Dittmann R, et al. Coexistence of filamentary and homogeneous resistive switching in Fe-doped $\mathrm{SrTiO}_{3}$ thin-film memristive devices. Adv Mater 2010, 22: 4819-4822.

[15] Rothschild A, Menesklou W, Tuller HL, et al. Electronic structure, defect chemistry, and transport properties of $\mathrm{SrTi}_{1-x} \mathrm{Fe}_{x} \mathrm{O}_{3-y}$ solid solutions. Chem Mater 2006, 18: 3651-3659.

[16] Steinsvik S, Bugge R, Gjønnes J, et al. The defect structufe OF $\mathrm{SrTi}_{1-x} \mathrm{Fe}_{x} \mathrm{O}_{3-y}(x=0-0.8)$ investigated by electrical conductivity measurements and electron energy loss spectroscopy (EELS). J Phys Chem Solids 1997, 58: 969-976.

[17] Vračar M, Kuzmin A, Merkle R, et al. Jahn-Teller distortion around $\mathrm{Fe}^{4+}$ in $\mathrm{Sr}\left(\mathrm{Fe}_{x} \mathrm{Ti}_{1-x}\right) \mathrm{O}_{3-\delta}$ from X-ray absorption spectroscopy, X-ray diffraction, and vibrational spectroscopy. Phys Rev B 2007, 76: 174107.

[18] Rodewald S, Fleig J, Maier J. Microcontact impedance spectroscopy at single grain boundaries in Fe-doped $\mathrm{SrTiO}_{3}$ polycrystals. J Am Ceram Soc 2001, 84: 521-530.

[19] Sundell PG, Björketun ME, Wahnström G. Thermodynamics of doping and vacancy formation in $\mathrm{BaZrO}_{3}$ perovskite oxide from density functional calculations. Phys Rev B 2006, 73: 104112 .

[20] Johnson KD, Dravid VP. Static and dynamic electron holography of electrically active grain boundaries in $\mathrm{SrTiO}_{3}$. Interface Sci 2000, 8: 189-198.

[21] Wang YG, Tang XG, Liu QX, et al. Ferroelectric and ferromagnetic properties of $\mathrm{SrTi}_{0.9} \mathrm{Fe}_{0.1} \mathrm{O}_{3-\delta}$ thin films. Solid State Commun 2015, 202: 24-27.

[22] Baker JN, Bowes PC, Long DM, et al. Defect mechanisms of coloration in Fe-doped $\mathrm{SrTiO}_{3}$ from first principles. Appl Phys Lett 2017, 110: 122903.

[23] Neaton JB, Ederer C, Waghmare UV, et al. First-principles study of spontaneous polarization in multiferroic $\mathrm{BiFeO}_{3}$. Phys Rev B 2005, 71: 014113.

[24] Hosokura T, Iwaji N, Nakagawa T, et al. (100)-oriented $\mathrm{SrTiO}_{3} / \mathrm{BaTiO}_{3}$ artificial superlattices fabricated by chemical solution deposition. Cryst Growth Des 2011, 11: 4253-4256.

[25] Carter E, Carley AF, Murphy DM. Evidence for $\mathrm{O}_{2}^{-}$radical stabilization at surface oxygen vacancies on polycrystalline $\mathrm{TiO}_{2}$. J Phys Chem C 2007, 111: 10630-10638.

[26] Caretti I, Keulemans M, Verbruggen SW, et al. Light-induced processes in plasmonic gold/ $/ \mathrm{TiO}_{2}$ photocatalysts studied by electron paramagnetic resonance. Top Catal 2015, 58: 776-782.
[27] Huang MH, Xia JY, Xi YM, et al. Study on photochromism in $\mathrm{SrTiO}_{3}$ :Fe ceramic powder. J Eur Ceram Soc 1997, 17: 1761-1765.

[28] Ehre D, Cohen H, Lyahovitskaya V, et al. X-ray photoelectron spectroscopy of amorphous and quasiamorphous phases of $\mathrm{BaTiO}_{3}$ and $\mathrm{SrTiO}_{3}$. Phys Rev B 2008, 77: 184106 .

[29] Yamashita T, Hayes P. Analysis of XPS spectra of $\mathrm{Fe}^{2+}$ and $\mathrm{Fe}^{3+}$ ions in oxide materials. Appl Surf Sci 2008, 254: 2441-2449.

[30] Perry NH, Kim N, Ertekin E, et al. Origins and control of optical absorption in a nondilute oxide solid solution: $\mathrm{Sr}(\mathrm{Ti}$, $\mathrm{Fe}) \mathrm{O}_{3-x}$ perovskite case study. Chem Mater 2019, 31: 1030-1041.

[31] Cooper D, Baeumer C, Bernier N, et al. Anomalous resistance hysteresis in oxide ReRAM: Oxygen evolution and reincorporation revealed by in situ TEM. Adv Mater 2017, 29: 1700212.

[32] Merino NA, Barbero BP, Eloy $\mathrm{P}$, et al. $\mathrm{La}_{1-x} \mathrm{Ca}_{x} \mathrm{CoO}_{3}$ perovskite-type oxides: Identification of the surface oxygen species by XPS. Appl Surf Sci 2006, 253: 1489-1493.

[33] Ghaffari M, Liu T, Huang H, et al. Investigation of local structure effect and X-ray absorption characteristics (EXAFS) of Fe (Ti) K-edge on photocatalyst properties of $\mathrm{SrTi}_{(1-x)} \mathrm{Fe}_{x} \mathrm{O}_{(3-\delta)}$. Mater Chem Phys 2012, 136: 347-357.

[34] Rana A, Lu HD, Bogle K, et al. Scaling behavior of resistive switching in epitaxial bismuth ferrite heterostructures. $A d v$ Funct Mater 2014, 24: 3962-3969.

[35] Ghaffari M, Shannon M, Hui H, et al. Preparation, surface state and band structure studies of $\operatorname{SrTi}_{(1-x)} \mathrm{Fe}_{(x)} \mathrm{O}_{(3-\delta)}(x=$ $0-1)$ perovskite-type nano structure by X-ray and ultraviolet photoelectron spectroscopy. Surf Sci 2012, 606: 670-677.

[36] Sahner K, Schönauer D, Moos R, et al. Effect of electrodes and zeolite cover layer on hydrocarbon sensing with p-type perovskite $\mathrm{SrTi}_{0.8} \mathrm{Fe}_{0.2} \mathrm{O}_{3-\delta}$ thick and thin films. J Mater Sci 2006, 41: 5828-5835.

[37] Bocquet, Fujimori, Mizokawa, et al. Electronic structure of $\mathrm{SrFe}^{4+} \mathrm{O}_{3}$ and related $\mathrm{Fe}$ perovskite oxides. Phys Rev B Condens Matter 1992, 45: 1561-1570.

[38] Du HC, Jia CL, Mayer J, et al. Atomic structure of antiphase nanodomains in Fe-doped $\mathrm{SrTiO}_{3}$ films. Adv Funct Mater 2015, 25: 6369-6373.

Open Access This article is licensed under a Creative Commons Attribution 4.0 International License, which permits use, sharing, adaptation, distribution and reproduction in any medium or format, as long as you give appropriate credit to the original author(s) and the source, provide a link to the Creative Commons licence, and indicate if changes were made.

The images or other third party material in this article are included in the article's Creative Commons licence, unless indicated otherwise in a credit line to the material. If material is not included in the article's Creative Commons licence and your intended use is not permitted by statutory regulation or exceeds the permitted use, you will need to obtain permission directly from the copyright holder.

To view a copy of this licence, visit http://creativecommons. org/licenses/by/4.0/. 